\title{
Electron holograms encoding amplitude and phase for the generation of arbitrary wavefunctions
}

Vincenzo Grillo, ${ }^{1,2,3}$ Ebrahim Karimi, ${ }^{4}$ Roberto Balboni ${ }^{5}$, Gian Carlo Gazzadi, ${ }^{1}$ Federico Venturi ${ }^{6}$, Stefano Frabboni, ${ }^{1,6}$ Jordan S Pierce $^{3}$, Benjamin $\mathrm{J} \mathrm{McMorran}^{3}$ and Robert W Boyd ${ }^{4,7}$

1. CNR-Istituto Nanoscienze, Centro S3, Via G. Campi 213/a, I-41125 Modena, Italy

2. CNR-IMEM Parco Area delle Scienze 37/A, I-43124 Parma, Italy

3. Department of Physics, University of Oregon, Eugene, 97403-1274 Oregon, USA

4. Department of Physics, University of Ottawa, 25 Templeton, Ottawa, Ontario, K1N 6N5 Canada

5. CNR-IMM Bologna, Via P. Gobetti 101, 40129 Bologna, Italy

6. Dipartimento FIM, Universitá di Modena e Reggio Emilia, Via G. Campi 213/a, I-41125 Modena, Italy

${ }^{7 .}$ Institute of Optics, University of Rochester, Rochester, New York 14627, USA

Since the introduction of vortex beams it has become clear that it is possible to structure the electron beams and produce arbitrary electron phase profiles [1][2]. We also demonstrated that the control over the phase in some cases like Bessel beams is sufficient to shape the overall amplitude profile for example by controlling the zeros of the electron wavefunction [3].

However in many cases the control of both amplitude and phase is demanded to engineer more structural properties of the electronic beam.

We report for the first time the electron holograms for phase and amplitude manipulation. The hologram we present is based on a modification of blazed phase holograms where the groove depth is modulated to produce the desired amplitude effect. The same philosophy can be applied to any initial groove profile. Calculations based on Fourier-Taylor expansion demonstrate the appropriate form of the transmission function has to be

$T=\exp (i M(x, y) \operatorname{Mod}(F(x, y)+2 \pi x, 2 \pi))$

with

$M(x, y)=1+\frac{1}{\pi} \operatorname{sinc} c^{-1}(A), \quad F(x, y)=P-\pi M$

where $\mathrm{A}$ and $\mathrm{P}$ are the aimed phase and amplitude distribution at the hologram plane, $\operatorname{Mod}(a, b)$ is the remainder of division of $a$ by $b$ and $\operatorname{sinc}^{-1}$ is the inverse of the function $\sin x / x$.

Fig 1 a,b show one example of FIB nanofabricated hologram encoding phase and amplitude information for a non-trivial beam. The aimed pattern calculated with the equation above is shown in fig 1a while the thickness map of the SiN membrane after FIB patterning is shown in fig $1 \mathrm{~b}$. The thickness measurement have been carried on by EFTEM in a JEOL 2010 equipped with LaB $_{6}$ emitter and operated at $200 \mathrm{keV}$. The aimed beam is given by the superposition of two Laguerre Gauss beams. If we indicate $L_{\ell, p}$ the Laguerre-Gauss with OAM quantum number $\ell$ and radial number $\mathrm{p}$, the aimed beam is

$\psi=\frac{\sqrt{2}}{2}\left(L_{-3,0}+L_{8,2}\right)$

This pattern has been chosen as example of complicated amplitude and phase modulation. The hologram has been introduced in a FEI-Tecnai F20T operated at $200 \mathrm{kV}$, in the sample position and the diffraction has been observed using low-mag diffraction mode.

Fig 1c shows the comparison between the experimentally generated intensity of the beam (left) and the 
aimed beam (right). In the simulated beam the intensity is proportional to image brightness and the hue is proportional to the phase.

It is worth remarking that the new holographic scheme, if compared to the original blazed hologram [4], presents a larger flexibility: however while phase-only Blazed holograms permits, at least in principle, to obtain a situation with only one diffracted beam, the present phase-amplitude hologram cannot eliminate residual intensity on all diffracted orders and in particular on the 0th order. An example in both experiment (above) and theory (Below) of $+/-1$ and $0^{\text {th }}$ diffraction order is shown in fig $1 \mathrm{~d}$.

\section{References:}

[1] J. Verbeeck, H. Tian, and P. Schattschneider, Nature 467, (2010) p 301.

[2] B. J. McMorran, A. Agrawal, I. M. Anderson, A. A. Herzing, H. J. Lezec, J. J. McClelland, and J. Unguris, Science 331, 192 (2011).

[3] V. Grillo, E. Karimi, G C Gazzadi, S Frabboni,M R. Dennis, and R W. Boyd Phys. Rev. X 4, 011013 (2014)

[4] V. Grillo, G C Gazzadi, E. Karimi, E Mafakheri,R W. Boyd, and S Frabboni Applied Physics Letters 104, 043109 (2014)
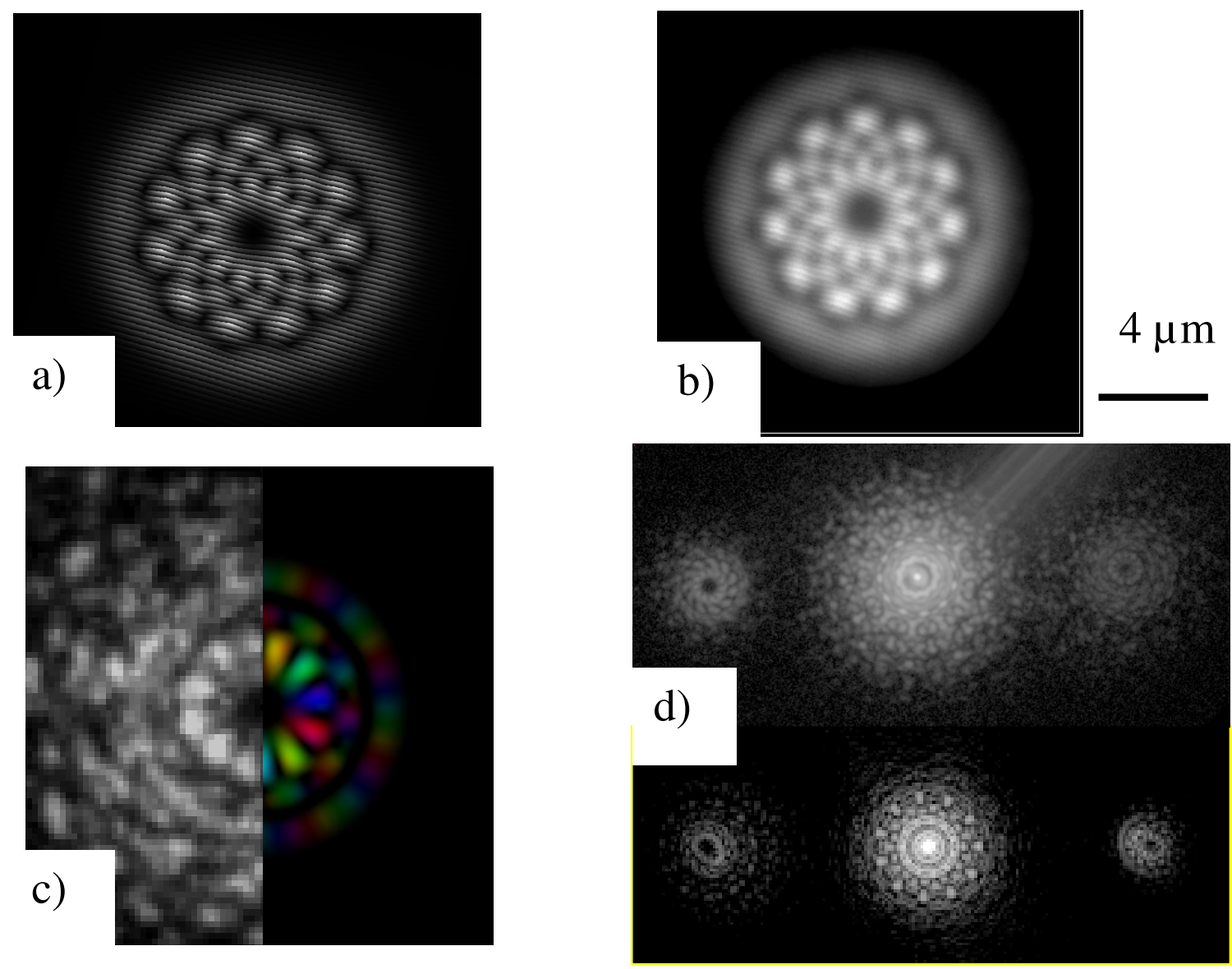

Figure 1. a) Aimed hologram pattern characterized by amplitude modulated blazed groove profile b) Experimental thickness map of the generated hologram c) comparison between experimental (left) and theoretical (right) beam. In the latter the phase has been highlighted by hue colorscale where hue is the phase. d) comparison of the full diffraction in experiment (above ) and simulations (below). 